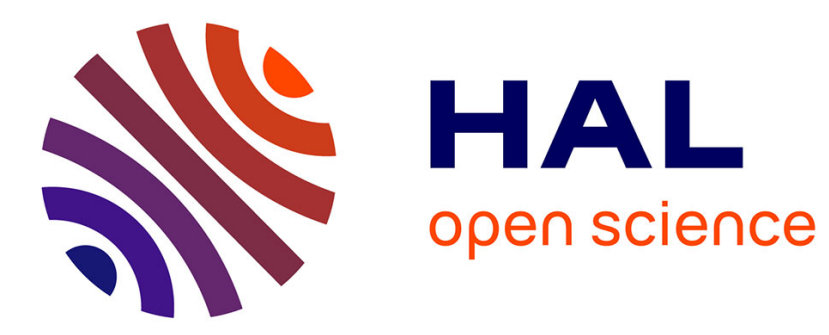

\title{
Dispositif de mesure de pouvoir thermoélectrique à très basse température
}

J. Chaussy, Ph. Gandit, A. Ravex

\section{To cite this version:}

J. Chaussy, Ph. Gandit, A. Ravex. Dispositif de mesure de pouvoir thermoélectrique à très basse température. Revue de Physique Appliquée, 1982, 17 (8), pp.499-502. 10.1051/rphysap:01982001708049900 . jpa-00245027

\section{HAL Id: jpa-00245027 https://hal.science/jpa-00245027}

Submitted on 1 Jan 1982

HAL is a multi-disciplinary open access archive for the deposit and dissemination of scientific research documents, whether they are published or not. The documents may come from teaching and research institutions in France or abroad, or from public or private research centers.
L'archive ouverte pluridisciplinaire HAL, est destinée au dépôt et à la diffusion de documents scientifiques de niveau recherche, publiés ou non, émanant des établissements d'enseignement et de recherche français ou étrangers, des laboratoires publics ou privés. 


\title{
Dispositif de mesure de pouvoir thermoélectrique à très basse température
}

\author{
J. Chaussy, Ph. Gandit et A. Ravex \\ Centre de Recherches sur les Très Basses Températures, C.N.R.S., \\ B.P. 166 X, 38042 Grenoble Cedex, France
}

(Reçu le 16 mars 1982, révisé le 13 mai 1982, accepté le 14 mai 1982)

\begin{abstract}
Résumé. - Nous décrivons une méthode de mesure du pouvoir thermoélectrique $S(T)$ à très basse température. L'exceptionnelle sensibilité d'un voltmètre continu développé dans notre laboratoire, utilisant un hacheur supraconducteur travaillant à la température de l'hélium, nous permet d'utiliser une technique différentielle donnant accès à une mesure directe de $S(T)$. Les résultats obtenus entre $10 \mathrm{mK}$ et $6 \mathrm{~K}$ avec divers alliages dilués or-fer nous permettent d'envisager dorénavant l'utilisation de thermocouples pour des mesures d'écart de températures $\Delta T$ de l'ordre de la dizaine de $\mu \mathrm{K}$ (chaleur spécifique ou conduction thermique) dans la gamme du milliKelvin.
\end{abstract}

\begin{abstract}
We describe a technique of measurement for thermoelectric power $S(T)$ at very low temperature. The very high sensitivity of a direct voltmeter developed in our laboratory using a superconducting chopper at helium temperature allows us to make a direct measurement of $S(T)$ by a differential technique. The results obtained between $10 \mathrm{mK}$ and $6 \mathrm{~K}$ with several $\mathrm{Au}-\mathrm{Fe}$ dilute alloys show that it is now possible to use thermocouple for temperature gradients measurements (specific heat or thermal conductivity) in the milliKelvin temperature range.
\end{abstract}

1. Introduction. - Les thermocouples sont abondamment utilisés comme thermomètres au-dessus de la température de l'hélium liquide car ils offrent un certain nombre de caractéristiques intéressantes : reproductibilité, grande sensibilité, simplicité d'utilisation et faible capacité calorifique. Malheureusement à basse température $(T<1 \mathrm{~K})$, leur sensibilité diminue rapidement puisque le pouvoir thermoélectrique des éléments tend vers zéro; les forces électromotrices parasites qui apparaissent le long du circuit de mesure deviennent dès lors non négligeables. Au moyen d'un voltmètre continu développé au laboratoire [1], nous transformons, par l'intermédiaire d'un hacheur supraconducteur à la température de l'hélium, le signal continu fournit par le thermocouple en un signal alternatif. Ce signal alternatif est également amplifié à la température de l'hélium. Nous nous affranchissons ainsi des signaux parasites continus mentionnés précédemment. D'autre part la très grande sensibilité de cet appareil de mesure (environ $10^{-15} \mathrm{~V}$ ) nous permet d'effectuer une mesure directe du pouvoir thermoélectrique dans la gamme du milliKelvin par une méthode différentielle. Nous avons ainsi pu mesurer jusqu'à $10 \mathrm{mK}$ le pouvoir thermoélectrique de thermocouples Au-Fe usuels et vérifier la possibilité d'utiliser de tels thermomètres pour des mesures de chaleur spécifique différentielle ou de conduction thermique.

2. Technique expérimentale. -2.1 MÉTHODE DE MESURE. - Généralement [2], le pouvoir thermoélectrique $S(T)$ est déterminé par une méthode intégrale : on mesure à vrai dire la force électromotrice $E\left(T_{R}-T\right)$ qui apparait aux bornes d'un thermocouple dont l'une des soudures est maintenue à une température de référence $T_{\mathrm{R}}$ (glace fondante, azote liquide, hélium liquide) et l'autre à une température $T$ variable. La variation avec la température du pouvoir thermoélectrique $S(T)$ est alors tirée de la relation

$$
E\left(T_{\mathrm{R}}-T\right)=\int_{T}^{T_{\mathrm{R}}} S(T) \mathrm{d} T .
$$

A très basse température $S(T)$ devient très faible, cette méthode ne permet plus d'en tirer une valeur avec suffisamment de précision.

Nous utilisons une méthode différentielle qui donne un accès-direct à $S(T)$. Les soudures du thermocouple sont liées thermiquement aux extrémités d'ui barreau de laiton. Un faible gradient thermique $\Delta T$ appliqué à ce barreau donne naissance aux bornes du thermocouple à une force électromotrice $V=S(\bar{T}) \Delta T, \bar{T}$ 
étant la température moyenne du barreau. Tout le problème expérimental de cette technique tient en la mesure du gradient $\Delta T$ qui doit être aussi faible que possible et de la tension $V$ qui est de l'ordre du picovolt pour un $\Delta T$ de quelques pour cent, dans la gamme du milliKelvin.

2.2 Mesure DU GRADIENT THERMiQue $\Delta T$. - Le montage utilisé (Fig. 1) est celui de la méthode classique à deux thermomètres et deux chauffages utilisée en conduction thermique. Un barreau de laiton sur lequel sont fixés deux thermomètres $T_{1}$ et $T_{2}$ est relié à l'une de ses extrémités à la boîte à mélange d'un réfrigérateur à dilution par l'intermédiaire d'une fuite thermique. Cette fuite nous permet de travailler entre $15 \mathrm{mK}$ et $7 \mathrm{~K}$ (limite imposée par la transition de fils de mesures supraconducteurs) sans désamorcer la dilution. L'élément résistif en constantan d'une jauge de contrainte $(360 \Omega)$, collée à l'autre extrémité du barreau permet de créer le gradient de température $\Delta T$ entre les deux thermomètres. Typiquement nous travaillons avec un $\Delta T / \bar{T}$ de l'ordre de $3 \%$ ce qui correspond à des puissances de chauffage de l'ordre de $3 \mathrm{nW}$ à $15 \mathrm{mK}$ et $0,6 \mathrm{~mW}$ à $7 \mathrm{~K}$. Un écran de cuivre à la température $\bar{T}$ limite les apports de puissance parasite par rayonnement sur le barreau. Le thermoélément $\mathrm{Au}-\mathrm{Fe}$ est collé à l'araldite ou à la graisse Apiezon $\mathrm{N}$ le long du barreau de laiton. Les deux extrémités du fil $\mathrm{Au}-\mathrm{Fe}$, torsadées avec les fils du second thermoélément, sont soudées à l'indium par ultrasons sur une pastille de silicium elle-même soudée à l'indium sur le laiton afin d'assurer l'isolation électrique du thermoélément

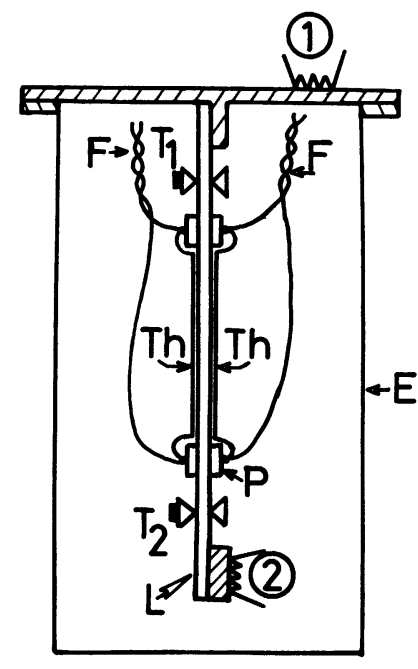

Fig. 1. - Montage expérimental : (1) et (2) = chauffages par jauges de contraintes (constantan $360 \Omega$ ); $\mathrm{T}_{1}$ et $\mathrm{T}_{2}=$ thermomètres silicium; $\mathrm{Th}=$ thermoéléments AuFe; $\mathrm{F}=$ fils supraconducteurs, $\mathrm{NbTi} ; \mathrm{E}=$ écran thermique en cuivre ; $\mathbf{P}=$ plaquettes de silicium $; \mathrm{L}=$ tige de laiton.

[Experimental arrangement : (1) and (2) = constantan strain gauge heaters $(360 \Omega) ; T_{1}$ and $T_{2}=$ silicon thermo-

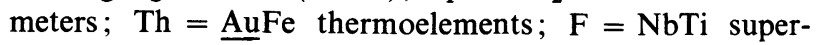
conducting wires $; \mathrm{E}=$ copper radiation shield $; \mathrm{P}=$ silicon slabs; $\mathbf{L}=$ brass rod.] tout en maintenant un bon contact thermique. Les deux thermomètres $T_{1}$ et $T_{2}$ sont des thermomètres au silicium dopé au bore qui présentent une grande sensibilité à très basse température [3] et qui sont étalonnés par rapport à la susceptibilité magnétique d'un CMN au-dessous de 1,4 K. Le thermomètre $\mathrm{T}_{2}$ seul détermine le gradient de température $\Delta T$ créé par le chauffage (2). La température du thermomètre $T_{1}$ est maintenue constante à l'aide d'une régulation pilotant le chauffage (1). Nous éliminons ainsi les erreurs liées à la détermination d'un écart de température au moyen de deux thermomètres. Ainsi nous mesurons en fait la conduction thermique du barreau de laiton (Fig. 3), ce qui nous permet de constater expérimentalement que nous n'avons aucun problème de thermométrie; en effet, toute erreur d'étalonnage ou tout mauvais contact thermique des thermomètres conduirait à une anomalie dans la loi linéaire de la conduction thermique $K(T)$ du laiton. De plus en ajustant les valeurs expérimentales à cette loi linéaire, nous obtenons un lissage des valeurs de $\Delta T$ qui diminue la dispersion sur la courbe $S(T)$.

2.3 Mesure DE LA TENSION $V$. - Les tensions mises en jeu aux températures les plus basses sont très faibles : en effet à $10 \mathrm{mK}$ le pouvoir thermoélectrique des éléments $\mathrm{Au}-\mathrm{Fe}$ est typiquement de $10^{-2} \mu \mathrm{V} / \mathrm{K}$ ce qui pour un $\Delta T / T$ de $1 \%$ donne une tension $V=10^{-12} \mathrm{~V}$. Il n'est pas envisageable de mesurer de telles tensions continues hors du cryostat car les forces électromotrices parasites créées le long du circuit électrique seraient rapidement supérieures au signal. Dans ce cas, on utilise généralement aujourd'hui un SQUID pour mesurer de telles tensions. Un des inconvénients de cet appareil qui à vrai dire est un magnétomètre est qu'il nécessite une impédance très faible pour le circuit de mesure. De plus, il n'est sensible qu'à une variation dynamique de température et ne voit pas un écart statique. Pour certaines applications des thermocouples, ces limitations peuvent s'avérer gênantes, en particulier si l'on cherche à diminuer la conduction thermique du thermoélément ou si l'on veut effectuer des mesures de conduction thermique. Le voltmètre développé au laboratoire ne présente pas cet inconvénient. Il s'agit d'un amplificateur à hacheur supraconducteur fonctionnant à l'hélium liquide. Le schéma électrique ainsi que le détail de la partie hacheur de cet appareil sont représentés en figure 2 . Il est réalisé à partir d'une couche mince supraconductrice dans laquelle est gravé le circuit du hacheur. Ce circuit se compose de quatre éléments supraconducteurs disposés en pont. Les éléments ont une résistance élevée à l'état normal. La transition de l'état supraconducteur à l'état normal est obtenue en chauffant les branches du pont par un rayonnement électromagnétique. Ce rayonnement est produit directement à l'intérieur du cryostat, au moyen de deux diodes électroluminescentes placées devant le circuit du hacheur, les deux diodes agissant chacune sur deux côtés opposés du pont. Les principaux avantages de ce 

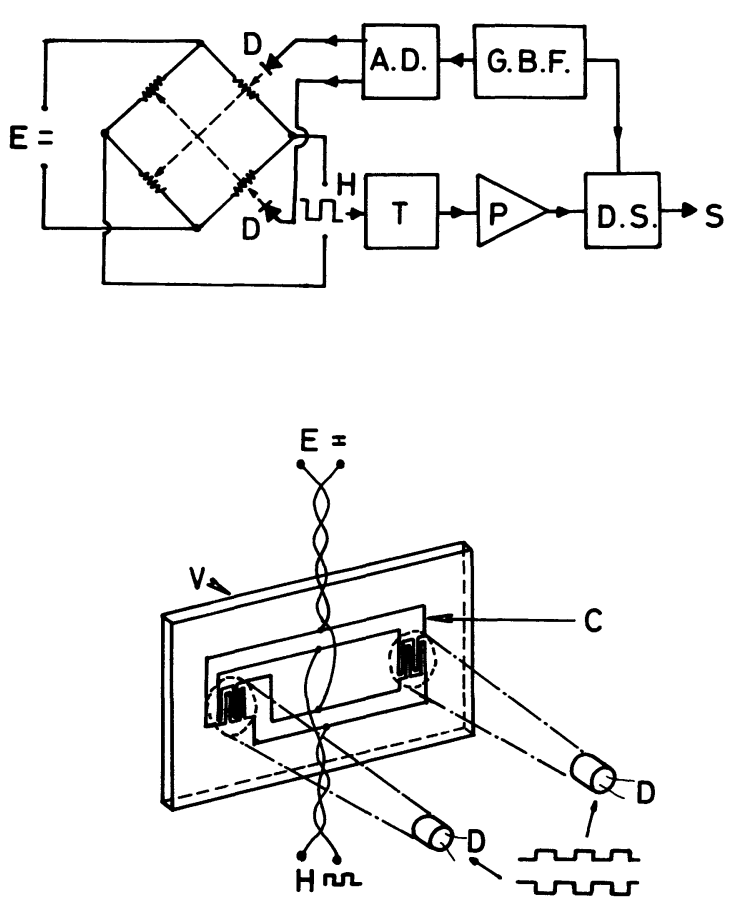

Fig. 2. - Schéma électrique du hacheur : $\mathrm{E}=$ entrée (signal continu) $\mathbf{V}=$ substrat de verre $; \mathrm{C}=$ circuit gravé dans une couche mince; $\mathrm{D}=$ diode électroluminescente ; $\mathbf{H}=$ signal haché $; \mathrm{S}=$ sortie signal $; \mathrm{T}=$ transformateur; $\mathbf{P}=$ préamplificateur à bas bruit $;$ D.S. = détection synchrone; G.B.F. = générateur basse fréquence ; A.D. = alimentation des diodes.

[Schematic view of the chopper : $\mathrm{E}=$ input (direct voltage); $\mathrm{V}=$ glass substrat $; \mathrm{C}=$ circuit etched from a thin film; $\mathrm{D}=$ light emitting diode $; \mathbf{H}=$ chopped voltage $; \mathrm{S}=$ output voltage $; \mathrm{T}=$ transformer $; \mathbf{P}=$ low noise preamplifier ; D.S. = phase sensitive detector; G.B.F. = oscillator; A.D. = current power supply.]

hacheur sont un bruit propre très faible (sensibilité $4 \times 10^{-15} \mathrm{~V}$, avec constante de temps de $1,7 \mathrm{~s}$ ), la possibilité d'utilisation de sources d'impédance interne élevée $(1 \mathrm{k} \Omega)$, une vitesse de commutation élevée (ce qui permet d'envisager un fonctionnement jusqu'à $1 \mathrm{kHz}$ ). En outre, cet appareil est peu encombrant et d'une grande simplicité d'utilisation.

3. Résultats expérimentaux. - Nous avons mesuré les deux types de thermocouples AuFe couramment utilisés en thermométrie à basse température :

- Au-Fe $0,07 \%$ atomique fourni par Leico Industrie, Inc. (New York), sous forme de fil de $80 \mu \mathrm{m}$ isolé au téflon;

- Au-Fe $0,03 \%$ atomique fourni par Johnson Matthey Limited (London), sous forme de fil de $80 \mu \mathrm{m}$ verni.

Comme second élément du thermocouple, nous avons utilisé du fil supraconducteur de niobium titane. Le principal avantage est que les supraconducteurs ont un pouvoir thermoélectrique nul, ainsi nous avons accès à une mesure absolue du pouvoị thermoélec-

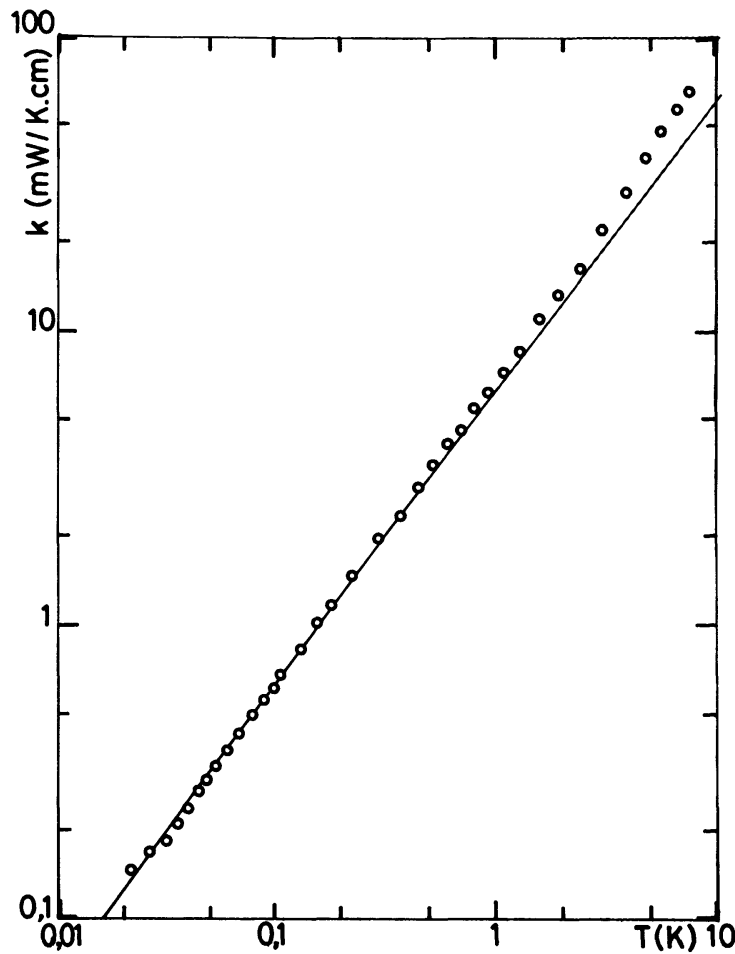

Fig. 3. - Conductivité thermique du laiton - la ligne pleine représente la contribution linéaire des électrons à basse température.

[Brass thermal conductivity - the straight line shows the low temperature electronic linear contribution.]

trique du seul élément Au-Fe. D’autre part, la faible conduction thermique des fils supraconducteurs élimine les problèmes d'apport de puissance parasite par conduction à l'échantillon.

Comme nous l'avons mentionné précédemment, nous effectuons une mesure de la conduction thermique du barreau de laiton. Sur la figure 3 , nous avons reporté les valeurs expérimentales obtenues pour la conductibilité thermique du laiton. Il a été vérifié que la contribution à la conduction thermique totale des fils $\mathrm{Au}-\mathrm{Fe}$ est négligeable (environ $0,16 \mu \mathrm{W} / \mathrm{K}$ à $100 \overline{\mathrm{mK}}$ à comparer à $24 \mu \mathrm{W} / \mathrm{K}$ pour le laiton). Nous observons qu'il n'apparaît aucune anomalie même aux plus basses températures par rapport à la loi de variation linéaire en température attendue pour un métal. Aux plus hautes températures $(T>2 \mathrm{~K})$, on voit apparaître la contribution en $T^{2}$ due aux phonons. Une analyse des résultatş expérimentaux suivant la loi $k=a T+b T^{2}$ donne par moindres carrés

$$
a=6,10 \mathrm{~mW} / \mathrm{K}^{2} \mathrm{~cm} \text { et } b=0,39 \mathrm{~mW} / \mathrm{K}^{3} \mathrm{~cm} \text {. }
$$

Cette loi permet la détermination, à partir de la puissance de chauffe $\dot{Q}$ et de la conduction de barreau $K=k s / l$, de l'écart de température $(\Delta T=\dot{Q} / K)$ utilisé pour le calcul du pouvoir thermoélectrique $(S=V / \Delta T)$. La précision de nos mesures sur $S$ est déterminée par les précisions obtenues d'une part sur $\Delta T$ et d'autre part sur la tension mesurée $V$. En 


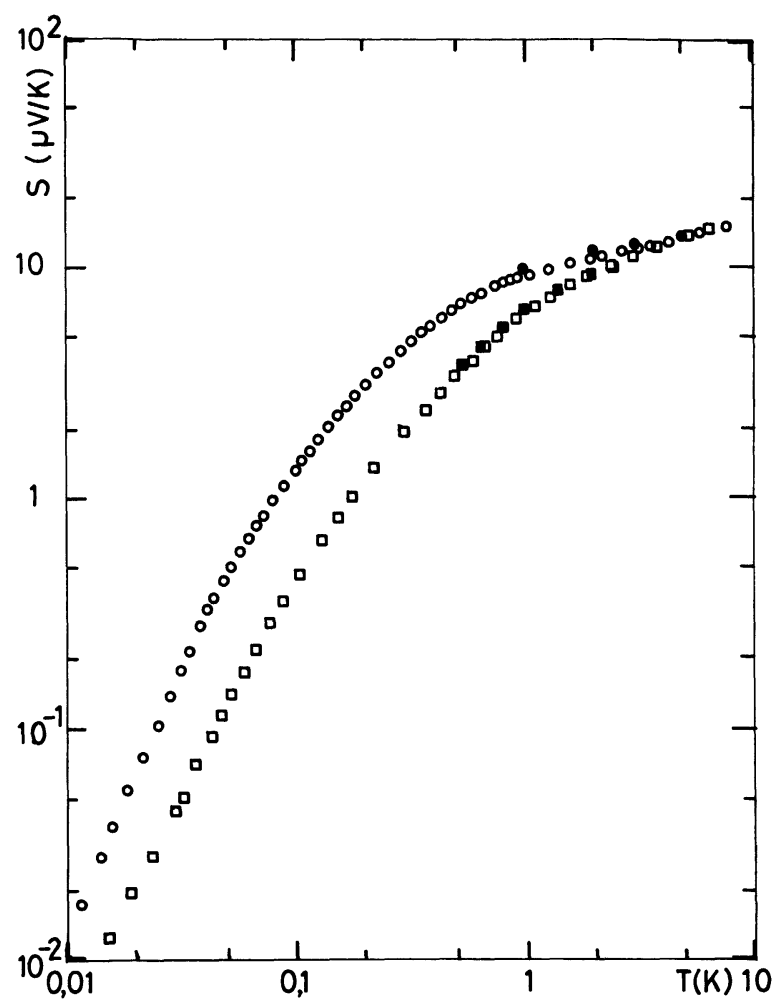

Fig. 4. - Pouvoir thermoélectrique d'alliages dilués $\mathrm{Au}-\mathrm{Fe}$ : ○ $0,03 \%$ atomique ( Berman et al., 1964); $\square \overline{0,07 \%}$ atomique ( $\square$ Rosenbaum, 1969).

[Thermoelectrical power of dilute alloys AuFe : 0 0.03\% (at.) (@ Berman et al., 1964); $\square 0.07 \%$ (at.)( $\square$ Rosenbaum, 1969).]

ce qui concerne le gradient de température, la précision est de l'ordre de $1 \%$ sur toute la gamme de température. Aux plus basses températures, la précision sur $V$ est du même ordre de grandeur, par contre, elle devient meilleure que le pour cent pour $T>100 \mathrm{mK}$.

En figure 4, nous avons reporté les pouvoirs thermoélectriques mesurés des deux éléments $\mathrm{Au}-\mathrm{Fe}$. $\mathrm{Au}$-dessus de $500 \mathrm{mK}$, nos valeurs expérimentales sont en très bon accord avec des mesures antérieures [2] qui étaient limitées à cette température par les problèmes liés à la mesure de la tension. D'autre part, dans cette région de température, les pouvoirs thermoélectriques des deux éléments sont voisins, indépendamment de la concentration en impureté de fer : le pouvoir thermoélectrique est dû à l'effet Kondo, interactions des impuretés magnétiques de fer avec la matrice d'or. Par contre, à basse température, la diminution de la concentration en fer augmente le pouvoir thermoélectrique qui est alors principalement limité par les interactions entre atomes de fer. Des mesures avec d'autres concentrations en fer sont en cours [4] pour vérifier cet effet et tenter d'obtenir des pouvoirs thermoélectriques supérieurs dans la gamme du milliKelvin.

4. Conclusion. - Nous avons montré qu'il est possible de mesurer directement avec une grande précision le pouvoir thermoélectrique de thermocouples usuels Au-Fe jusqu'à 10 milliKelvin. Ceci nous permet d'envisager des mesures différentielles de chaleur spécifique dans cette gamme de température en utilisant les thermocouples en détecteurs de zéro ce qui nous affranchirait d'un étalonnage précis. La haute résolution du voltmètre à hacheur dans les conditions expérimentales $\left(10^{-12} \mathrm{~V}\right.$ à $20 \mathrm{mK}$ pour une impédance de source de l'ordre de $5 \times 10^{-2} \Omega$ ) nous permet de conserver une grande sensibilité aux plus basses températures malgré le faible pouvoir thermoélectrique des éléments $\mathrm{Au}-\mathrm{Fe}\left(5 \times 10^{-2} \mu \mathrm{V} / \mathrm{K}\right)$. Nous pouvons ainsi mesurer un écart de température de $20 \mu \mathrm{K}$ à $20 \mathrm{mK}$.

\section{Bibliographie}

[1] Y. NG. ToNG, Thèse Docteur-Ingénieur, Grenoble (1980), appareil développé au CNRS-CRTBT Grenoble qui a fait l'objet d'un brevet ANVARCNRS.

[2] Berman, R., Brock, J. C. F., Huntley, D. J., Cryogenics 4 (1964) 233.

Rosenbaum, R. L., Rev. Sci. Instrum. 40 (1969) 577.

MaC Donald, D. K. C., Pearson, W. B., TempleTON, I. M., Proc. R. Soc. A 266 (1962) 161.
Chaussy, J., Guessous, A., Mazuer, J., Rev. Sci. Instrum. 52 (1981) 1721.

[3] Frossati, G., Imbert, D., Peccoud, L., Thoulouze, D., Waksmann, B., Proc. LT 14 Conf., M. Krusius Ed. 4 (1975) 206.

[4] Chaussy, J., Gandit, Ph., Matho, K., Ravex, A., $J$. Low Temp. Phys. 49 no 1/2 (1982) (à paraître). 